\title{
Breaking van der Waals molecules with magnetic fields
}

\author{
R.V. Krems \\ Harvard-MIT Center for Ultracold Atoms, Department of Physics, Harvard University and \\ Institute for Theoretical Atomic, Molecular and Optical Physics, Harvard-Smithsonian Center for \\ Astrophysics, Cambridge, MA 02138 \\ Electronic mail: rkrems@cfa.harvard.edu
}

(November 5, 2018)

\begin{abstract}
It is demonstrated that weakly bound van der Waals complexes can dissociate in a magnetic field through coupling between the Zeeman levels. The Zeeman predissociation process is shown to be efficient and it can be controlled by external magnetic fields.
\end{abstract}

PACS numbers: 34.50.-s, 32.80.Pj, 32.60.+i, 34.20.6j

Manipulating dynamics of molecules with external fields has long been a sought-after goal of experimental and theoretical research. Several groups have studied elastic and inelastic collisions of ultracold alkali atoms in the presence of Feshbach scattering resonances [1-6]. It was found that the probabilities of elastic collisions and inelastic energy transfer undergo dramatic changes near Feshbach resonances. The Feshbach resonances can be tuned by bias magnetic fields so that atom-atom ultracold collisions can be controlled. Ultracold molecules have been created by linking ultracold atoms with magnetic fields [7]. The creation of molecules at subKelvin temperatures has opened up possibilities for controlling molecular interactions with electric fields [8]. External fields break the isotropy of space and may induce couplings between electronic states otherwise uncoupled [9]. Forbidden electronic transitions may thus become allowed in an external field and the probabilities of the electronic transitions can be controlled by the strength of the external field [9].

In this work we study predissociation of weakly bound van der Waals complexes containing atoms with non-zero electronic orbital angular momentum in a magnetic field when transitions to lower magnetic levels release enough energy to break the van der Waals bond. The predissociation is due to coupling between different Zeeman energy levels of the complex. We show that the Zeeman predissociation dynamics can be efficient and the molecules, while stable at zero magnetic field, quickly decompose in a magnetic field.

Figure 1 illustrates the idea of the Zeeman predissociation. Consider a van der Waals complex of an open-shell atom (A) with non-zero electronic orbital angular momentum and a closed-shell atom or molecule (B) in a magnetic field. The AB complex can be described by a set of coupled potential energy curves correlating with different Zeeman energy levels of atom A. At zero magnetic field, the Zeeman energy levels are degenerate and the complex is stable. An external magnetic field splits the Zeeman energy levels and bound states of the complex may be higher in energy than some of the Zeeman levels of the separated A and B. The AB molecule can then dissociate through transitions to these Zeeman levels. The predissociation lifetime depends on the strength of the coupling between the Zeeman levels. 
If atom $\mathrm{A}$ is in an electronic $S$-state, the A-B interaction is independent of spin and the Zeeman energy levels are not coupled but they are if atom A is in a state with non-zero orbital angular momentum. If B is a closed-shell atom, the A-B interaction potential can be expanded in a Legendre series [10]

$$
V_{\mathrm{AB}}(\mathbf{R}, \hat{r})=\sum_{\lambda} \frac{4 \pi}{2 \lambda+1} V_{\lambda}(R) \sum_{m_{\lambda}} Y_{\lambda m_{\lambda}}^{*}(\hat{R}) Y_{\lambda m_{\lambda}}(\hat{r}),
$$

where $R$ is the interatomic distance, $\hat{R}$ is the unit vector with the direction of $\mathbf{R}, \hat{r}$ denotes collectively the position vectors of the open-shell electrons and the expansion coefficients $V_{\lambda}(R)$ can be expressed in terms of the non-relativistic interaction potentials of the $\mathrm{AB}$ molecule [11]. The vector sum of the electronic orbital $\mathbf{L}$ and spin $\mathbf{S}$ angular momenta gives the total electronic angular momentum $\mathbf{J}_{\mathrm{a}}$ of atom A. The asymptotic $R=\infty$ states of the $\mathrm{AB}$ complex in a magnetic field are characterized by the rotational angular momentum $l$ of $\mathrm{AB}$ and the projections $m_{l}$ and $M$ of $\mathbf{l}$ and $\mathbf{J}_{\mathrm{a}}$ on the magnetic field axis, respectively [9]. The magnetic field couples the states of different total angular momentum $J_{\mathrm{a}}$, but not the $M$-states, so that the Zeeman transitions are induced by the couplings between states $\left|J_{\mathrm{a}} M l m_{l}\right\rangle$ and $\left|J_{\mathrm{a}}^{\prime} M^{\prime} l^{\prime} m_{l}^{\prime}\right\rangle$. The integrals over the wavefunctions $\left|J_{\mathrm{a}} M l m_{l}\right\rangle$ with the interaction potential (1) can be evaluated analytically to yield [9]

$$
\begin{gathered}
\left\langle J_{\mathrm{a}}(L S) M l m_{l}\left|V_{\mathrm{AB}}\right| J_{\mathrm{a}}^{\prime}(L S) M^{\prime} l^{\prime} m_{l}^{\prime}\right\rangle=\sum_{\lambda} V_{\lambda} \sum_{m_{\lambda}}(-1)^{S+J_{\mathrm{a}}+J_{\mathrm{a}}^{\prime}+\lambda+m_{\lambda}-m_{l}-M} \\
{\left[(2 L+1)(2 L+1)\left(2 J_{\mathrm{a}}+1\right)\left(2 J_{\mathrm{a}}^{\prime}+1\right)(2 l+1)\left(2 l^{\prime}+1\right)\right]^{1 / 2} \times} \\
\left\{\begin{array}{ccc}
L & J_{\mathrm{a}} & S \\
J_{\mathrm{a}}^{\prime} & L & \lambda
\end{array}\right\}\left(\begin{array}{ccc}
J_{\mathrm{a}} & \lambda & J_{\mathrm{a}}^{\prime} \\
-M & m_{\lambda} & M^{\prime}
\end{array}\right)\left(\begin{array}{ccc}
l & \lambda & l^{\prime} \\
-m_{l} & -m_{\lambda} & m_{l}^{\prime}
\end{array}\right)\left(\begin{array}{ccc}
L & \lambda & L \\
0 & 0 & 0
\end{array}\right)\left(\begin{array}{lll}
l & \lambda & l^{\prime} \\
0 & 0 & 0
\end{array}\right),
\end{gathered}
$$

where symbols in parentheses and curly braces are $3 j$ - and $6 j$-symbols. Eq. (2) establishes that the Zeeman energy levels are coupled by the anisotropic part of the interaction potential (1). Because the interaction of atoms with non-zero $L$ depends strongly on the relative orientation of $\hat{R}$ and $\hat{r}$, the Zeeman couplings are significant in the van der Waals complexes with such atoms.

To form an idea of lifetimes for the Zeeman predissociation in complexes with open-shell atoms, we analyzed the predissociation of the $\mathrm{He}-\mathrm{O}\left({ }^{3} P\right)$ molecule in a magnetic field. The ground state of oxygen is ${ }^{3} P_{2}$. It splits into five Zeeman levels with energies approximately equal to $3 B \mu_{B} M / 2$, where $\mu_{B} \approx 0.47 \mathrm{~cm}^{-1} /$ Tesla. This suggests that the $\mathrm{HeO}$ molecules bound by $11.2 \mathrm{~cm}^{-1}$ or less may undergo the Zeeman predissociation at $B=4$ Tesla. The ground state of the He- $\mathrm{O}\left({ }^{3} \mathrm{P}\right)$ complex is characterized by two non-relativistic potentials of $\Sigma$ and $\Pi$ symmetry [12]. Although the well depths of the potentials are only 9.4 and $20.9 \mathrm{~cm}^{-1}$, respectively, they support several bound states. To identify the predissociating states, we computed the $S$-matrix eigenphase sum for $\mathrm{He}-\mathrm{O}\left({ }^{3} P\right)$ collisions on a dense grid of energies below the $M=+2$ threshold. The theory of the scattering calculations has been described in our preceding paper [9]. The eigenphase sum is obtained by summing the inverse tangents of the eigenvalues of the $K$-matrix [13]. The eigenphase sum rises with energy by $\pi$ near an isolated resonance [14].

Figure 2 shows the eigenphase sum for $\mathrm{He}-\mathrm{O}\left({ }^{3} \mathrm{P}\right)$ as a function of energy and external magnetic field. When oxygen is in the ${ }^{3} P_{2}$ state, there are no couplings between the states 
of odd and even partial waves $l$ ( $c f$. Eq. 2). Five Zeeman levels corresponding to $J_{\mathrm{a}}=2$ and three states of the orbital angular momentum, $l=0,2$ and 4 , are included in these calculations with the fixed total angular momentum projection $M+m_{l}=2$. The spin-orbit excited states of oxygen are separated from the $J_{\mathrm{a}}=2$ state by 158.1 and $226.6 \mathrm{~cm}^{-1}$, so they were neglected. The number of resonances becomes larger and the resonances broaden with increasing magnetic field. The lifetimes of the metastable states can be computed from the energy derivative of the eigenphase sum at the positions of the resonances [13]. To make sure that our calculations are correct, we computed the derivatives both by the finite difference method and analytically from piecewise Chebyshev approximations of the eigenphase sum. The positions and lifetimes of the resonances of Fig. 2 are summarized in Table 1. The lifetimes decrease by almost two orders of magnitude as the magnetic field increases from 0.1 to 3 Tesla.

The rise of the eigenphase sum in Fig. 2 is due to the predissociating states or shape resonances in open channels. To verify that we do observe the Zeeman predissociation, we computed the bound states of the $\mathrm{O}\left({ }^{3} P_{2}\right)$-He complex as a function of the magnetic field. To do that, we diagonalized the Hamiltonian of the $\mathrm{O}\left({ }^{3} P_{2}\right)$-He complex at zero magnetic field using a Hund's case (e) representation for the atomic wave functions [15] and a Fourier basis discrete variable representation (DVR) of Colbert and Miller [16] for the radial part of the wave function. The bound states are characterized by the value of total angular momentum $\mathbf{J}=\mathbf{J}_{\mathrm{a}}+\mathbf{l}$, parity $\epsilon=(-1)^{L+l}$ and a quantum number $s$ describing the stretching motion of the nuclei (see Table 2). To confirm the accuracy of our results, we repeated the calculation in the Hund's case (c) representation of the molecular functions. The total Hamiltonian was then diagonalized in the fully uncoupled representation (2) as a function of the magnetic field strength. This procedure yields converged results for the bound energy levels and the predissociating states with lifetime $>10^{-9}$ sec, which was verified by the close coupling calculations. As the lifetime of the predissociating states becomes smaller than $10^{-9} \mathrm{sec}$, the DVR calculations converge very slowly and the close coupling calculation was used to obtain the magnetic field dependence of the predissociating states at high magnetic fields. The close coupling solution at high magnetic fields was matched with the DVR results at low fields to provide the magnetic field dependence of the energy levels in the full interval of magnetic fields considered. This allowed us to identify three resonances in Fig. 1 and Table 1 with the quantum numbers $J_{\mathrm{a}}$ and $s$. The fourth resonance is a shape resonance.

When the strength of the magnetic field increases, more Zeeman levels become energetically accessible and the dissociation can occur through transitions to more states. The energy gap between the initial and final energy levels is larger in higher magnetic fields and the wave packet escapes faster over the centrifugal barriers in the outgoing channels. At the same time, more bound states become open for predissociation in higher magnetic fields (see Fig. 1). Thus the number of metastable states and the dissociation efficiency increase with increasing field strength.

The lifetimes of the bound states decaying through the Zeeman predissociation are small (see Table 1) indicating that the Zeeman levels in He-O $\left({ }^{3} P\right)$ are strongly coupled. Yet, it should be expected that the degree of anisotropy is smaller in the HeO complex than in most other van der Waals complexes containing atoms with non-zero $L$. The strength and anisotropy of interaction of oxygen with other rare gas atoms are larger [12]. The degree of anisotropy is also larger in complexes containing D-state atoms [17]. 
If $\mathrm{A}$ is an open-shell atom and $\mathrm{B}$ is a $\Sigma$-state diatomic molecule, the angular expansion of the A-B interaction potential is analogous to that of two diatomic molecules [18]. It can be written in the form [19]

$$
\begin{gathered}
V_{\mathrm{AB}}\left(\mathbf{R}, \hat{r}_{\mathrm{A}}, \mathbf{r}_{\mathrm{B}}\right)=(4 \pi)^{3 / 2} \sum_{\lambda_{\mathrm{A}} \lambda_{\mathrm{B}} \lambda} V_{\lambda_{\mathrm{A}} \lambda_{\mathrm{B}} \lambda}\left(R, r_{\mathrm{B}}\right) \\
\times \sum_{m_{\lambda_{\mathrm{A}}} m_{\lambda_{\mathrm{B}}} m_{\lambda}}\left(\begin{array}{ccc}
\lambda_{\mathrm{A}} & \lambda_{\mathrm{B}} & \lambda \\
m_{\lambda_{\mathrm{A}}} & m_{\lambda_{\mathrm{B}}} & m_{\lambda}
\end{array}\right) Y_{\lambda_{\mathrm{A}} m_{\lambda_{\mathrm{A}}}}\left(\hat{r}_{\mathrm{A}}\right) Y_{\lambda_{\mathrm{B}} m_{\lambda_{\mathrm{B}}}}\left(\hat{r}_{\mathrm{B}}\right) Y_{\lambda m_{\lambda}}(\hat{R}),
\end{gathered}
$$

where $\hat{r}_{\mathrm{A}}$ is the same as $\hat{r}$ in Eq. (1) and $\hat{r}_{\mathrm{B}}$ describes the orientation of molecule $\mathrm{B}$ in the space-fixed coordinate frame. The Zeeman energy levels are coupled by the matrix elements between states $\left|N M_{N} J_{\mathrm{a}} M l m_{l}\right\rangle$ and $\left|N^{\prime} M_{N}^{\prime} J_{\mathrm{a}}^{\prime} M^{\prime} l^{\prime} m_{l}^{\prime}\right\rangle$, where $N$ is the rotational angular momentum quantum number and $M_{N}$ is the projection of $\mathbf{N}$. The matrix elements of the interaction potential can be evaluated using the Wigner-Eckart theorem as follows

$$
\begin{array}{r}
\left\langle N M_{N} J_{\mathrm{a}} M l m_{l}\left|V_{\mathrm{AB}}\right| N^{\prime} M_{N}^{\prime} J_{\mathrm{a}}^{\prime} M^{\prime} l^{\prime} m_{l}^{\prime}\right\rangle=\sum_{\lambda_{\mathrm{A}} \lambda_{\mathrm{B}} \lambda} V_{\lambda_{\mathrm{A}} \lambda_{\mathrm{B}} \lambda} \\
\times \sum_{m_{\lambda_{\mathrm{A}}} m_{\lambda_{\mathrm{B}}} m_{\lambda}}\left(\begin{array}{ccc}
\lambda_{\mathrm{A}} & \lambda_{\mathrm{B}} & \lambda \\
m_{\lambda_{\mathrm{A}}} & m_{\lambda_{\mathrm{B}}} & m_{\lambda}
\end{array}\right)(-1)^{S+J_{\mathrm{a}}+J_{\mathrm{a}}^{\prime}+\lambda_{\mathrm{A}}-m_{l}-M-M_{N}} \\
{\left[(2 L+1)(2 L+1)\left(2 J_{\mathrm{a}}+1\right)\left(2 J_{\mathrm{a}}^{\prime}+1\right)(2 l+1)\left(2 l^{\prime}+1\right)(2 N+1)\left(2 N^{\prime}+1\right)\right]^{1 / 2}} \\
{\left[\left(2 \lambda_{\mathrm{A}}+1\right)\left(2 \lambda_{\mathrm{B}}+1\right)(2 \lambda+1)\right]^{1 / 2}\left\{\begin{array}{ccc}
L & J_{\mathrm{a}} & S \\
J_{\mathrm{a}}^{\prime} & L & \lambda_{\mathrm{A}}
\end{array}\right\}\left(\begin{array}{ccc}
J_{\mathrm{a}} & \lambda_{\mathrm{A}} & J_{\mathrm{a}}^{\prime} \\
-M & m_{\lambda_{\mathrm{A}}} & M^{\prime}
\end{array}\right)\left(\begin{array}{ccc}
l & \lambda & l^{\prime} \\
-m_{l} & m_{\lambda} & m_{l}^{\prime}
\end{array}\right)} \\
\times\left(\begin{array}{ccc}
L & \lambda_{\mathrm{A}} & L \\
0 & 0 & 0
\end{array}\right)\left(\begin{array}{ccc}
l & \lambda & l^{\prime} \\
0 & 0 & 0
\end{array}\right)\left(\begin{array}{ccc}
N & \lambda_{\mathrm{B}} & N^{\prime} \\
-M_{N} & m_{\lambda_{\mathrm{B}}} & M_{N}^{\prime}
\end{array}\right)\left(\begin{array}{ccc}
N & \lambda_{\mathrm{B}} & N^{\prime} \\
0 & 0 & 0
\end{array}\right)
\end{array}
$$

Eq. (4) shows that in addition to the anisotropy of interaction due to relative rotation of vectors $\hat{r}_{\mathrm{A}}$ and $\hat{R}$, the Zeeman transitions can be induced by the anisotropy of interaction arising from relative rotation of vectors $\hat{r}_{\mathrm{A}}$ and $\hat{r}_{\mathrm{B}}$. While the Zeeman transitions in atomatom systems described by Eq. (1) must always be accompanied by changes of the orbital angular momentum projection $m_{l}$, the Zeeman transitions in atom-molecule collisions may occur without any change of $m_{l}$. The orientation of the electronic momentum $\mathbf{J}_{\mathrm{a}}$ may be transferred to the rotational angular momentum $\mathbf{N}$ of the molecule. The interaction between the Zeeman levels will therefore be generally stronger in complexes of open-shell atoms with molecules.

In summary, we have demonstrated that weakly bound van der Waals complexes containing atoms with non-zero electronic orbital angular momentum can dissociate in a magnetic field through coupling between the Zeeman energy levels. The lifetimes for the Zeeman predissociation are small at laboratory moderate magnetic fields. They are similar to the lifetimes for rotational predissociation of triatomic van der Waals complexes such as $\mathrm{Ar}-\mathrm{HCl}$ [13]. Unlike ro-vibrational or electronic predissociation, the Zeeman predissociation can be controlled by an external magnetic field.

I would like to thank Alex Dalgarno for encouragement and useful comments on the manuscript and Sture Nordholm for hospitality during a visit to Göteborg University where this work was initiated. This work was supported by the Harvard-MIT Center for Ultracold Atoms and the Institute for Theoretical Atomic, Molecular and Optical Physics at the Harvard-Smithsonian Center for Astrophysics. 


\section{TABLES}

TABLE I. Positions $\left(E_{\mathrm{r}}\right.$ in $\left.\mathrm{cm}^{-1}\right)$ and lifetimes $\left(\tau_{\mathrm{r}}\right.$ in sec) of the resonances of Fig. 2. The energy is referred to the $\left(J_{\mathrm{a}}, M=+2\right)$ dissociation limit.

\begin{tabular}{ccccc}
\hline \hline$B$, Tesla & $J$ & $s$ & $E_{\mathrm{r}}$ & $\tau_{\mathrm{r}}$ \\
\hline 0.1 & & & -0.003 & $1.14 \times 10^{-9}$ \\
& 2 & 1 & -0.298 & $3.63 \times 10^{-9}$ \\
0.3 & & -0.131 & $3.41 \times 10^{-10}$ \\
& & & & \\
& 3 & 0 & -1.914 & $1.91 \times 10^{-8}$ \\
& 2 & 1 & -0.648 & $3.04 \times 10^{-9}$ \\
& & & -0.348 & $5.31 \times 10^{-11}$ \\
& & & & $4.33 \times 10^{-9}$ \\
& 2 & 0 & -4.023 & $8.54 \times 10^{-10}$ \\
& 3 & 0 & -2.814 & $1.84 \times 10^{-10}$ \\
& 2 & 1 & -1.248 & $4.00 \times 10^{-11}$ \\
& & & -0.403 & $1.11 \times 10^{-10}$ \\
& & & & $4.49 \times 10^{-11}$ \\
& 2 & 0 & -5.180 & $4.10 \times 10^{-11}$ \\
& 3 & 0 & -3.344 & $3.00 \times 10^{-11}$ \\
\hline \hline
\end{tabular}

TABLE II. Bound energy levels $\left(E_{r}\right.$ in $\left.\mathrm{cm}^{-1}\right)$ of the $\mathrm{O}\left({ }^{3} P_{2}\right)$-He complex at zero magnetic field. The energy is referred to the dissociation limit.

\begin{tabular}{llcc}
\hline \hline$J$ & $s$ & $\epsilon$ & $E_{r}$ \\
\hline 1 & 0 & +1 & -0.701 \\
& & & \\
2 & 0 & -1 & -3.170 \\
2 & 0 & +1 & -3.007 \\
2 & 1 & -1 & -0.176 \\
& & & -1.622 \\
3 & 0 & +1 & -0.947 \\
3 & 0 & -1 &
\end{tabular}




\section{FIGURES}
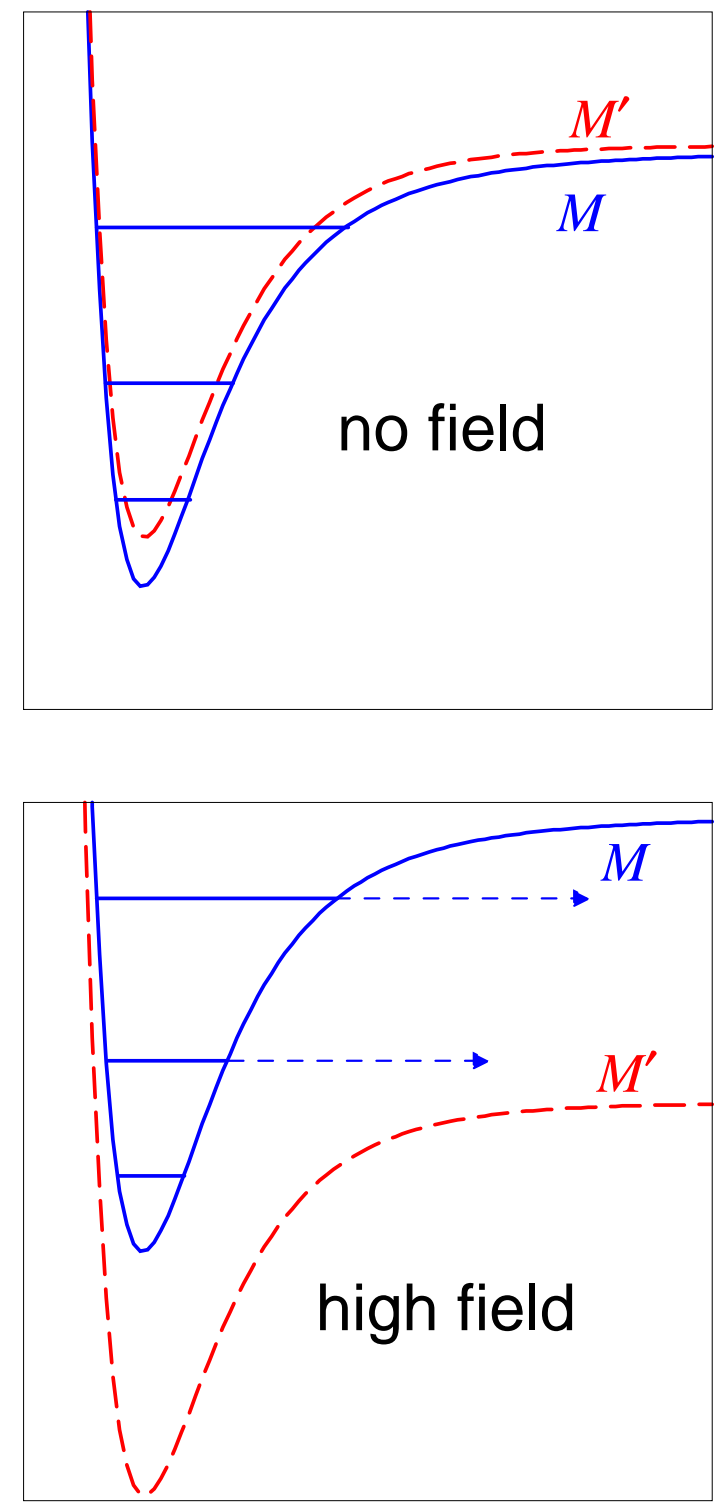

FIG. 1. Zeeman predissociation 

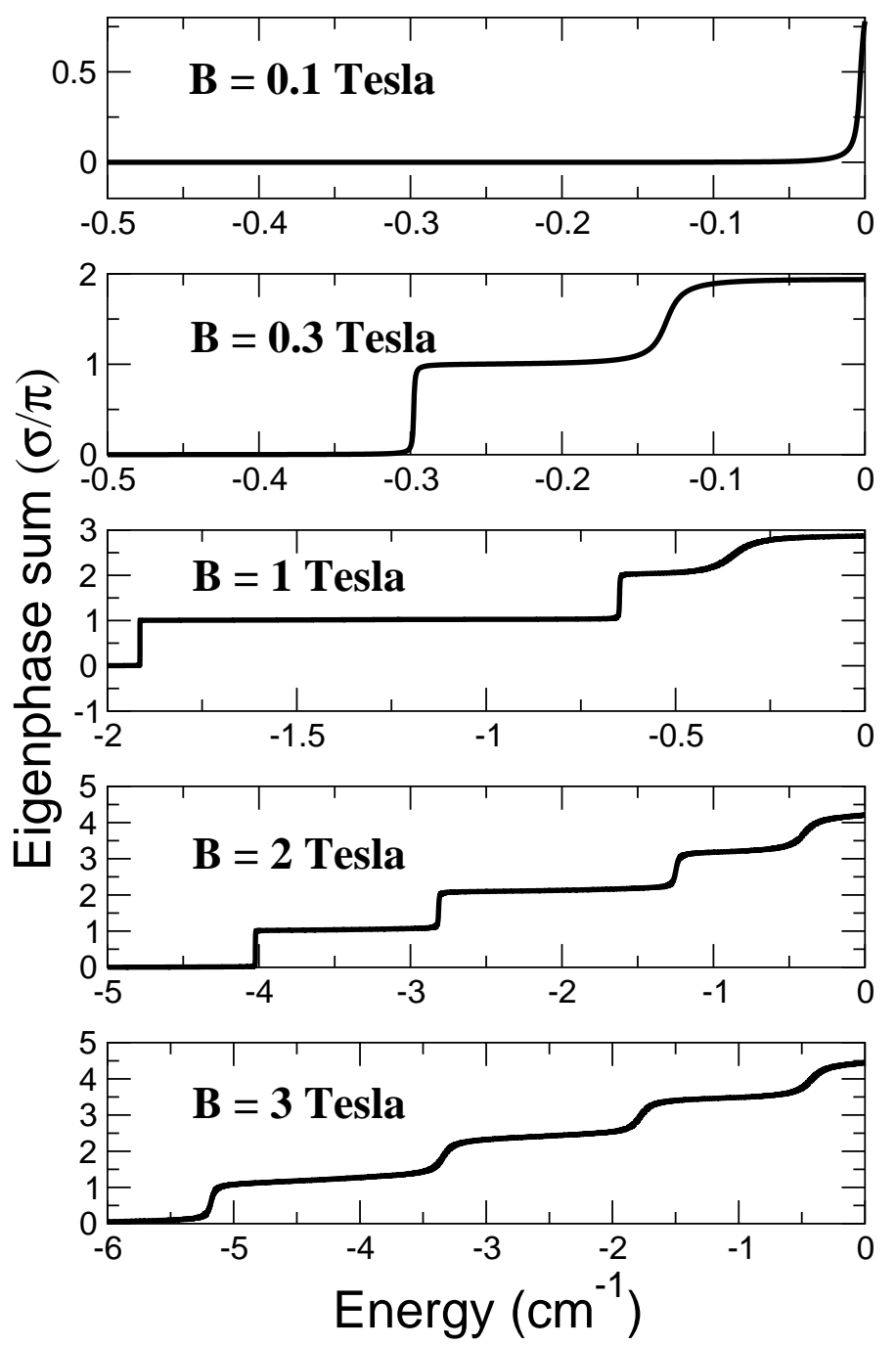

FIG. 2. $S$-matrix eigenphase sum of $\mathrm{He}-\mathrm{O}\left({ }^{3} P\right)$ in a magnetic field. The energy is referred to the $M=+2$ threshold. 


\section{REFERENCES}

[1] J. Stenger, S. Inouye, M. R. Andrews, H. J. Miesner, D. M. Stamper-Kurn, and W. Ketterle, Phys. Rev. Lett. 82, 2422 (1999).

[2] A. Marte, T. Volz, J. Schuster, S. Durr, G. Rempe, E. G. M. van Kempen, and B. J. Verhaar, Phys. Rev. Lett. 89, 283202 (2002).

[3] J. L. Roberts, N. R. Claussen, S. L. Cornish, and C. E. Wieman, Phys. Rev. Lett. 85, $728(2000)$.

[4] S. Jochim, M. Bartenstein, G. Hendl, J. H. Denschlag, R. Grimm, A. Mosk, and M. Weidemuller, Phys. Rev. Lett. 89, 273202 (2002).

[5] T. Loftus, C. A. Regal, C. Ticknor, J. L. Bohn, and D. S. Jin, Phys. Rev. Lett. 88, 173201 (2002).

[6] F. H. Mies, E. Tiesinga, and P. S. Julienne, Phys. Rev. A 61, 022721 (2000).

[7] C. A. Regal, C. Ticknor, J. L. Bohn, and D. S. Jin, Nature 424, 47 (2003).

[8] A. V. Avdeenkov and J. L. Bohn, Phys. Rev. Lett. 90, 043006 (2003).

[9] R.V. Krems and A. Dalgarno, Phys. Rev. A 68, 013406 (2003).

[10] R. H. G. Reid and A. Dalgarno, Phys. Rev. Lett. 22, 1029 (1969).

[11] V. Aquilanti and G. Grossi, J. Chem. Phys. 73, 1165 (1980).

[12] R.V. Krems, A.A. Buchachenko, M.M. Szczesniak, J. Klos, and G. Chalasinski, J. Chem. Phys. 116, 1457 (2002).

[13] C. J. Ashton, M. S. Child, and J. M. Hutson, J. Chem. Phys. 78, 4025 (1983).

[14] P.G. Burke, Adv. At. Mol. Opt. Phys. 4, 173 (1968); A. U. Hazi, Phys. Rev. A 19, 920 (1979).

[15] A. Carrington, C. A. Leach, A. J. Marr, A. M. Shaw, M. R. Viant, J. M. Hutson, and M. M. Law, J. Chem. Phys. 102, 2379 (1995).

[16] D. T. Colbert and W. H. Miller, J. Chem. Phys. 96, 1982 (1992).

[17] This discussion excludes transition metal atoms. The interaction anisotropy in complexes with transition metal atoms may be suppressed due to the peculiar character of their electronic structure.

[18] M.-L. Dubernet and J. M. Hutson, J. Chem. Phys. 101, 1939 (1994).

[19] P.E.S. Wormer and A. van der Avoird, J. Chem. Phys. 81, 1929 (1984); A. van der Avoird and G. Brocks, J. Chem. Phys. 87, 5346 (1987). 